\title{
Thermo-flow Analysis of Cylinder with Crossed Splitter Plates with a Characteristics-based Scheme
}

\author{
S. E. Razavi ${ }^{1}$, T. Adibi ${ }^{2}$ and H. Hassanpour ${ }^{3 *}$ \\ ${ }^{1}$ Department of Mechanical Engineering, University of Tabriz, Tabriz, Iran \\ ${ }^{2}$ Department of Mechanical Engineering, University of Bonab, Bonab, Iran \\ ${ }^{3}$ Department of Automotive Engineering, Iran University of Science and Technology, Tehran, Iran \\ E-mail: ${ }^{1}$ razavi@tabrizu.ac.ir, ${ }^{2}$ tohidadibi@ubonab.ac.ir, ${ }^{3}$ h hassanpur@alumni.iust.ac.ir
}

Received 15 December 2020, Revised 11 February 2021, Accepted 18 March 2021

\begin{abstract}
In the present work, the laminar flow through a circular cylinder with two crossed splitter plates is analyzed. The characteristic-based method has been used along with the unstructured grid. The current research has been done to detect the proper conditions according to the geometrical parameters in which the optimal heat transfer is taking place. Geometric control parameters are the angle of splitter plates (theta) and the ratio of length of the splitter plate to cylinder radius $(\mathrm{n}=\mathrm{L} / \mathrm{D})$. It was found that the use of a two-branched splitter plate is not wise in Reynolds number less than 100 due to its insignificant effect in flow properties. In angle $30^{\circ}$ between two plates, the least drag force is witnessed with respect to other angles. Application of double branched splitter with angles more than $60^{\circ}$ is not recommended, which will increase the total drag significantly. Since the splitter plate increases, the overall heat transfer from the cylinder and splitter set is enhanced. Minimum drag over the cylinder, and maximum convection drop from it is taken place when the dimension length is 0.75 . Between dimensionless lengths 1.25 and 1.5 , the Nusselt number oscillates with least amplitude and such behavior is also observed when two splitters are $60^{\circ}$ apart.
\end{abstract}

\section{Keywords: Double splitter plate, incompressible flow, Navier-stokes equations, Nusselt number}

\section{Introduction}

The flow past circular has been an issue of many studies so far, and this is because it has many practical functions in the industry, such as the study of heat exchangers, bridges, risers, Chimneys. The undesirable phenomena of vortex shedding and broad wake region downstream cause significant drag force and the reduction in heat transfer. Various attempts that have been made include both passive and active control systems. One of the simple passive ways to accomplish such enhancements will be to use the so-called splitter plates. The splitter plate creates a streamlined postponement of the circular tube, and in terms of heat transfer. However, splitter plate may decrease the amount of convection heat transfer since it also acts like a fin, the overall mixed conduction, convection overweighs heat transfer from the bare cylinder. Therefore, these plates enhance the heat transfer. Different numerical studies were done for incompressible thermo-flows to survey the other ways to improve heat transfer rates. Adibi et al. simulated thermo-flow using nano-fluids to enhance heat transfer rate[1]. Malik et al.[2] studied two-dimensional incompressible flow along with a stretching cylinder. Nusselt number is calculated in different cases. Similarity transformation was used to solve the governing equations. Bayat and Rahimi[3] simulated two-dimensional incompressible thermos-flow over a cylinder. The SIMPLE algorithm was used. Nusselt number and drag coefficient are obtained in different conditions. Different aspects of flow past cylinder are studied by Gu et al. [4], a wind tunnel for flow past cylinder with a splitter plate. They examined ten splitter plates with the length ratio (length of the splitter to cylinder diameter) varying from 0.5 to 6 . However, an easily rotatable splitter plate increases the average lift force towards the plate's deflected side. Igbalajobi et al. [5] investigated the effect of wake mounted splitter on the flow past cylinder using a low-speed wind tunnel. A significant drop in the average drag coefficient was found for the circular cylinder. They understood that the splitter plate was more effective in suppressing the Karman vortices for the smaller-aspect-ratio cylinders. Boisaubert and Texier [6], employing reliable tracer visualizations, investigated the splitter plate influence on the primary near-wake creation stages of a semi-circular cylinder geometry. The primary vortices are pushed with time to splitter plate trailing edge. Shukla et al. [7] studied a hinged-splitter plate behindhand the cylinder. The experiments showed that the splitter plate fluctuations rise with $\mathrm{Re}$, and reach a saturation largeness level at greater Re. The results demonstrated that the splitter plate length to cylinder diameter ratio $(\mathrm{L}=\mathrm{D})$ is crucial in determining the oscillations' character and magnitude. Reazvan Abdi et al. [8] investigated the fluid forces acting on a three cases including the cylinder with one, two or three rigid splitter plates attached at its rear surface were considered and the location of horizontal plates (attachment angle) was varied between $0^{\circ}$ and $90^{\circ}$ in a laminar flow regime while using a passive control strategy. They results shows that the location and the number of the plates have crucial effects on the wake control and increasing the number of splitter plates from one to two symmetric parallel plates led to a reduction in drag force, vortex shedding frequency and fluctuation of lift force. Shaoshi Dai et al. [9] were performed a systematic study to determine the optimal conditions and optimal splitter-plate 
dimensions for the suppression of vortex shedding from circular cylinders and minimize the fluctuating loads on the cylinder. Their results indicate that splitter plates provide a practical method for vortex suppression at high Reynolds numbers, and the degree of suppression can be maximized by optimal geometric configuration. Dong-Lai Gao et al. [10] set a short splitter-plate onto the upstream side of a circular cylinder to modify its incoming flow conditions and the wake-vortex patterns attenuate the aerodynamic forces. Their results show that the vortical structures rolled up on the upstream splitter plate and flow separation change the incoming flow of the cylindrical model and drag reduction is generally realized, but fluctuating-lift suppression needs to be careful, also the control effectiveness and the surrounding flow topologies obtained by PIV system help to uncover the mechanism of the passive upstream-plate control. Tiwari et al. [11] had carried out a numerical investigation to survey the $\mathrm{Nu}$ in flow with and without fins. A reduction in the wake size in comparison to the circular tube is observed. Overall, there is an enhancement in heat transfer for flow over the cylinder with a splitter plate compared with the case without a splitter plate. Akili et al. [12] controlled the flow over a perpendicular cylinder located in water by a splitter plate inserted at several places downstream of the cylinder. The splitter plate at the two-dimensional location, no effect of the plate was observed. Xinyu et al. [13] simulated twodimensional incompressible flow over a circular cylinder with a splitter numerically. Results showed that vortex shedding is observed when the splitter length is more extensive than four times the cylinder's diameter. The maximal lift coefficient is at the splitter length is about two times the cylinder's diameter. Sahu et al. [14] simulated a two-dimensional flow over the cylinder with a splitter by the finite element method. The dimension of the splitter is $3.5 \mathrm{D}$, and the Reynolds number is considered 150. Ghiasi et al. [15] investigated the influence of the active and passive ways on the flow and temperature fields to find an optimum situation. This combination results in heat transfer increment and drag reduction. Results showed that fluctuation in changed frequencies was complex. Upper frequencies result in more heat transfer. Mishra et al. [16] studied the laminar flow over a circular cylinder by a viscoelastic thin plate by the FEM. The results presented that careful tuning of the damping may be effectively employed to enhance power output for energy extraction applications or to suppress flow-induced vibration when it is detrimental to the structure. Chauhan et al. [17] considered flow over a cylinder with splitter experimentally for $R e=485$. Results showed that the drag coefficient and the Strouhal number decrease with an increase in splitter plate length. Assi and Bearman [18] investigated flow over a cylinder with three different splitter-plates considering plate length and plate porosity variations. Ghassabi and Kahrom [19] studied a thermos-flow over a square-cylinder experimentally. They performed their experiment for Reynolds number 5130. They found the optimum geometry. Among various papers dealing with the splitter plates, seldom is there one working on a two-branch splitter plate. This new kind of passive control device consists of two thin plates on the cylinder's trailing edge [20]. By varying the angle between two plates $\varphi$ and the ratio of the length of the splitter to the radius of the cylinder $\mathrm{n}$, different flow patterns, and Nusselt distributions are obtained. Therefore, we perform numerical analysis to find out the influence of several parameters on heat transfer factors. Finally, the effect of splitter presence on Nusselt number oscillations is shown and discussed. Since this bifurcated splitter plate has been simulated for the first time, it can be used in several practical problems, such as behind heat exchanger tubes, to enhance the heat transfer while keeping the drag to a minimum.

The present study results show the effect of two rigid separator plates on the drag coefficient and Nusselt number. This research can be used with changes in various industries such as automobile manufacturing, aircraft manufacturing, shipbuilding, and heat exchangers. An in-house FORTRAN 90 code has been written and developed by the authors, which implement the novel characteristic-based flux treatment. The novelty of this work could be expressed as following; Implementation of proposed characteristic-based scheme and verification of its robustness and performance in rather complicated flow patterns such as the present case (crossed splitter plates), and Investigation of thermo-flow behavior over crossed splitter plates in heat transfer enhancement.

\section{Methodology}

To investigate the flow around the cylinder with and without separator plates and the effect of the angle between them, Problem expression and problem-solving method can be seen in the flowchart Figure 1. Also, a two-dimensional and straightforward model according to Figure 2 and the initial data according to Table 1 have been considered. To study the mesh independence of the model for analyzing the fluid dynamics of the flow using the finite element method, the meshing of the flow region is shown in Figure 3.

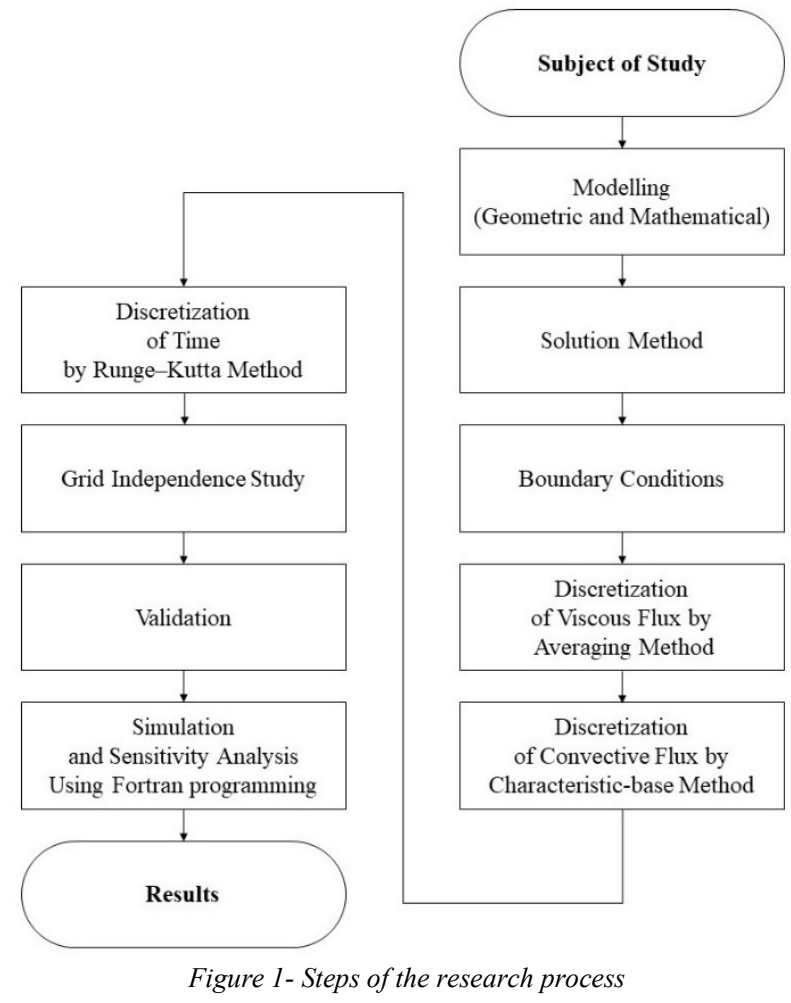

As shown in Figure 3, the flow zone mesh is clustered in sections close to the wall with larger dimensions and smaller numbers. In the area around the cylinder and separator plates with very fine and high-density clustering for high accuracy. The number of meshes in the flow zone from the lowest to the highest is between 300 and 35,000. 


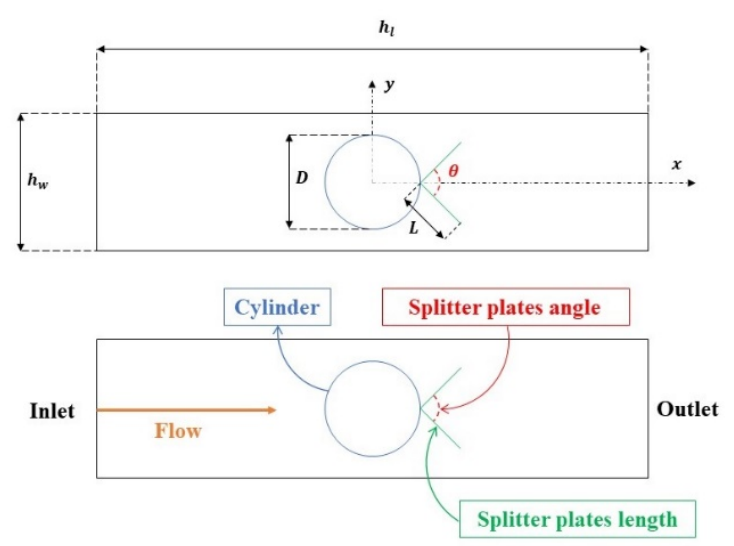

Figure 2- Scheme and geometry of the model

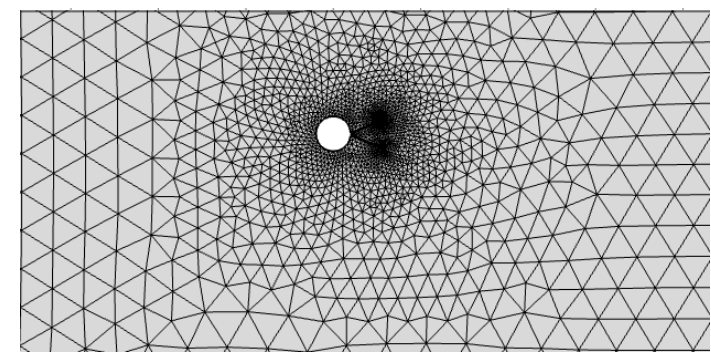

Figure 3. An enlarged view of the computational grid

Table 1- Numerical values for Input data of model

\begin{tabular}{ccc}
\hline Parameter & Quantity & Unit \\
\hline$v$ & 1 & $\mathrm{~m} / \mathrm{s}$ \\
$D$ & 10 & $\mathrm{~cm}$ \\
$L$ & $2.5-4.5$ & $\mathrm{~cm}$ \\
$\theta$ & $0-150$ & degree \\
$P_{\text {out }}$ & 1 & $\mathrm{~atm}$ \\
$T_{\text {in }}$ & 300 & $\mathrm{k}$ \\
$T_{\text {cyl }}$ & 400 & $\mathrm{k}$ \\
\hline
\end{tabular}

Navier-Stokes equations for incompressible and twodimensional viscous steady fluid are derived in eq. (1) [21];

$$
\begin{aligned}
& \frac{\partial u}{\partial x}+\frac{\partial v}{\partial y}=0 \\
& \frac{\partial u}{\partial t}+u \frac{\partial u}{\partial x}+v \frac{\partial u}{\partial y}= \\
& -\frac{1}{\rho} \frac{\partial p}{\partial x}+v\left(\frac{\partial^{2} u}{\partial x^{2}}+\frac{\partial^{2} u}{\partial y^{2}}\right) \\
& \frac{\partial v}{\partial t}+u \frac{\partial v}{\partial x}+v \frac{\partial v}{\partial y}= \\
& -g-\frac{1}{\rho} \frac{\partial p}{\partial y}+v\left(\frac{\partial^{2} v}{\partial x^{2}}+\frac{\partial^{2} v}{\partial y^{2}}\right) \\
& \frac{\partial T}{\partial t}+u \frac{\partial T}{\partial x}+v \frac{\partial T}{\partial y}= \\
& \frac{1}{\rho C}\left(u \frac{\partial p}{\partial x}+v \frac{\partial p}{\partial y}\right)+\frac{k}{\rho C_{p}}\left(\frac{\partial^{2} T}{\partial x^{2}}+\frac{\partial^{2} T}{\partial y^{2}}\right)+\frac{\mu}{\rho C_{p}} \Phi \\
& \Phi=\left(\frac{\partial u}{\partial y}+\frac{\partial v}{\partial x}\right)^{2}+2\left(\left(\frac{\partial u}{\partial x}\right)^{2}+\left(\frac{\partial v}{\partial y}\right)^{2}\right)
\end{aligned}
$$

Where $u$ and $v$ are fluid velocity in $x$ and $y$ direction, $p$ is the pressure, $T$ is temperature, $\rho$ is density, $\mu$ is static viscosity, $C_{P}$ is Specific heat capacity, and $\Phi$ is the dissipation term. Two types of boundary conditions are necessary to solve steady Navier-Stokes's equations. The flow conditions in the first place include specified velocity conditions at the inlet and specified pressure at the outlet.
The cylinder well and its splitters are considered solid walls. In thermal conditions, a Dirichlet type condition is exerted on boundary inlet, cylinder, and splitter plates [22]. Other walls of the boundary bear the Neuman insulation condition. No-slip condition is used for temperature and velocity of flow on the wall. Temperature and velocity of Inlet flow and pressure of outlet flow have specific numerical data.

$u_{\text {wall }}=0, v_{\text {wall }}=0, T_{\text {wall }}=T_{0}$,

$u_{\text {inlet }}=u_{0}, v_{\text {inlet }}=0, T_{\text {inlet }}=T_{1}, p_{\text {outlet }}=0$.

Numerical simulations are done using a FORTRAN code that authors write. The characteristic-based scheme introduced by the authors is used for convective fluxes[23, 24]. For viscous fluxes, the averaging method on the secondary cells is used[25]. The Fifth-order Runge-Kutta method is used for time marching[26].

\section{Results}

Because of the lack of sufficient data on the two branches splitter plate, we compare the data attained from the present model to that of the bare cylinder by setting the L/D ratio equal to zero and therefore produce the bare cylinder situation. The comparison is made between data attained from the ongoing model on a cylinder by a single branch splitter plate. The latter diagram will be a particular case to our two problems, one in which the angle $\theta$ is held zero. As it is seen from Figure 4, consonance exists between present data and that of Churchill [27], which proves the reliability of this model. The obtained drag coefficient in this current work is compared with Razavi et al. [28] in Figure 4.

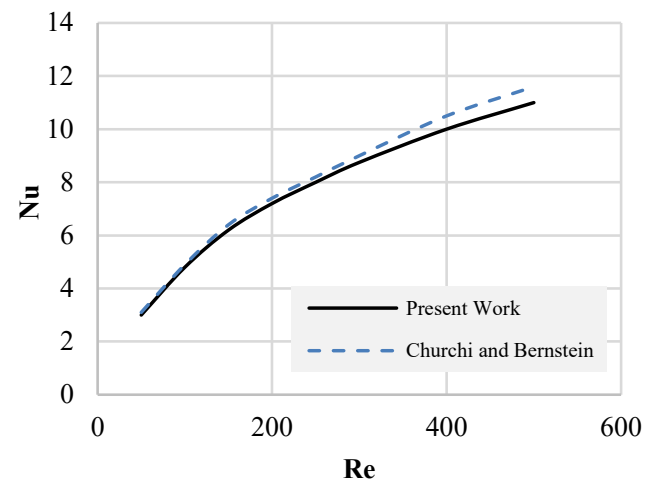

Figure 4. Comparison between Nusselt number diagrams for bare cylinder attained from the present model and Churchill and Bernstein [21] results

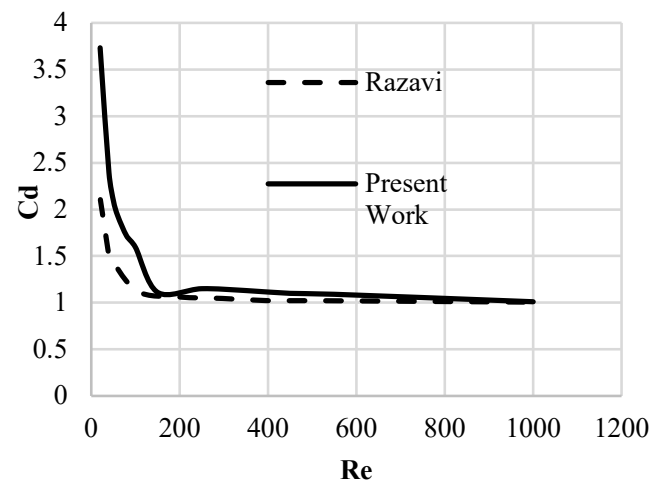

Figure 5 Comparison among obtained drag coefficient in present work with that of Razavi et al. [28] 


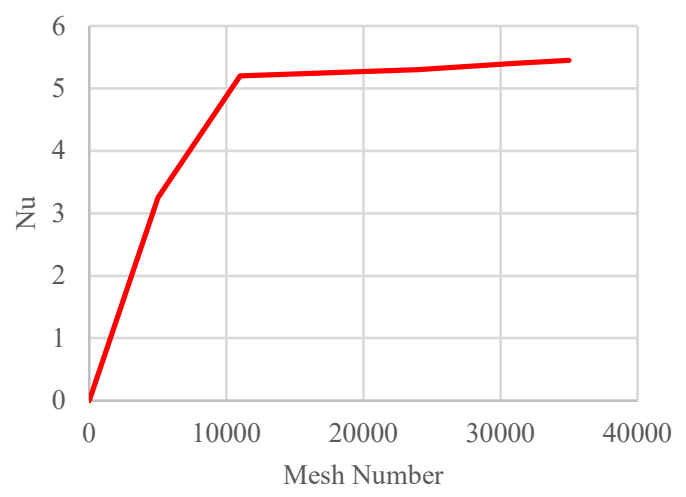

Figure 6. G rid independence (mean Nusselt number for different grid numbers)

Flow visualization for bare cylinder and cylinder with double branch splitter plate with different angles are shown in Figure 7. As seen in Figure 7, flow characteristics as vortices for bare cylinder and cylinders with splitter are different.

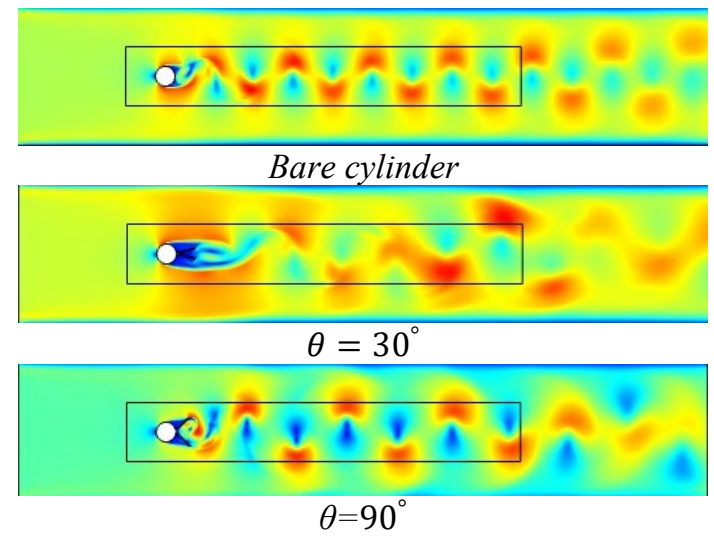

Figure 7. Flow visualization for bare cylinder and cylinder with double branch splitter plate with different angles

The drag coefficient for the cylinder with double branch splitter to that of the bare cylinder (which is denoted as $\mathrm{Cd} 0$ ) for different Reynolds and various angles among two plates is shown in Figure 8. It is noteworthy to be kept in mind, though splitters are present; the numerator of this drag fraction only accounts for drag force on the cylinder [29]. In other words, this fraction is inspecting the effect of the presence of splitter on the cylinder alone and not cylinder and splitter assembly.

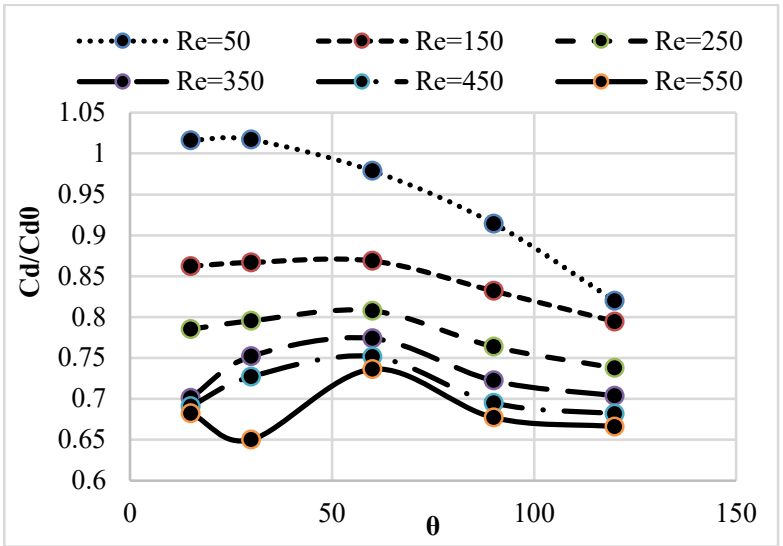

Figure 8. The ratio of the drag coefficient on cylinder for cylinder and splitter plate assembly to that of bare cylinder
When the Reynolds number is about 50 , the diagram is close to unity, which means the splitter plate is of no use in low Reynolds no matter what the angle among two plates is. The reason is that the vortices have not yet taken their persuasive and robust form. By increasing the splitters' angle, the plates will stand in the position more normal to the flow, and downstream of the circular cylinder in the region between two plates; the flow becomes slower. Where the velocity is low, the pressure change between the sides of the circular cylinder will decrease; This results in drag reduction. That is why the diagrams drastically drop when $\theta$ goes more than $60^{\circ}$. This diagram shows the ratio of drag coefficient property of cylinder with splitters to that of the bare cylinder; this drop of diagrams mean when $\theta$ goes more than $60^{\circ}$ the splitter has relieved its corresponding cylinder from much drag force.

When $\theta$ is about $30^{\circ}$ the cylinder is less available by the first strong vortex. A vortex creates a low-pressure area by its nature, which means the closer the vortex is to the cylinder, the more pressure difference for the two parts of the flow. With this idea in mind, the least drag is expected to happen when $\theta$ is about $30^{\circ}$. And vice versa, and as we saw in the earlier section when $\theta$ is about $60^{\circ}$ The first strong vortex is closest to the cylinder, which makes a peak in drag force. As it is clear from the diagram for $\mathrm{Re}=550$, when Reynolds is high enough for vortices to show their role in the flow, $\theta=30^{\circ}$ is the best angle according to drag and $\theta=$ $60^{\circ}$ is worst.

In Figure 9, it is claimed that the effect of the splitter's normal direction on the flow and distance of $1^{\text {st }}$ strong vortex will play an influential role. When the Reynolds number is about 50, the vortex regime is so weak, and the splitter's presence makes no sense to the Nusselt number, and the diagram is close to unity. When the $\Theta$ rises, the plates' position seems to be more normal to the flow direction, debilitating the flow near downstream, which means less motion downstream. This will cause less convection heat transfer. That is why all the diagrams in Figure 9 maintain their general decreasing tone on all Reynolds. As the Reynolds number gets high, vortices become more robust, and where $R e=550$, a peak and a valley will show up. The valley belongs to $\theta=30^{\circ}$ where the cylinder is less available the first strong vortex downstream. Hence, the peak is for $\theta=60^{\circ}$ that the vortex regime is like bare cylinder.

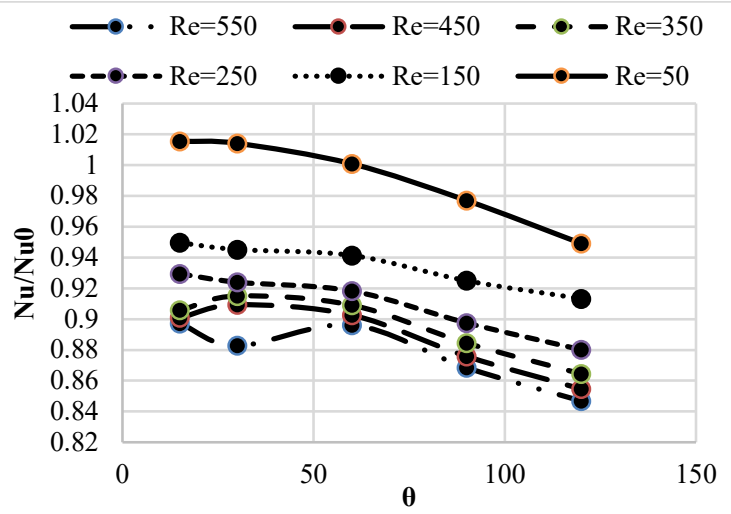

Figure 9. Nusselt Ratio between cylinder bearing double branch splitter plate and bare cylinder.

In Figure 10, in various Reynolds numbers, increasing the $\Theta$ made the flow to strike harder to the splitter and in turn, increase the total drag in Greater Reynolds numbers. This 
means all diagrams must keep their increasing tone, which is clear in Figure 10. At $\theta=30^{\circ}$ the minimum is witnessed the total drag reduction. This drags reduction gets strong as the Reynolds grow (showing up the significant amount of about $40 \%$ less than bare cylinder when $\mathrm{Re}=550$ ). Around $\theta=$ $60^{\circ}$ the ratio of total drag coefficient over cylinder and splitter assembly to that of bare cylinder commences rising significantly. We could deduce that $\theta=30^{\circ}$ is the best option for $\Theta$ according to total drag conditions. Angles more than $60^{\circ}$ are not appropriate to be used.

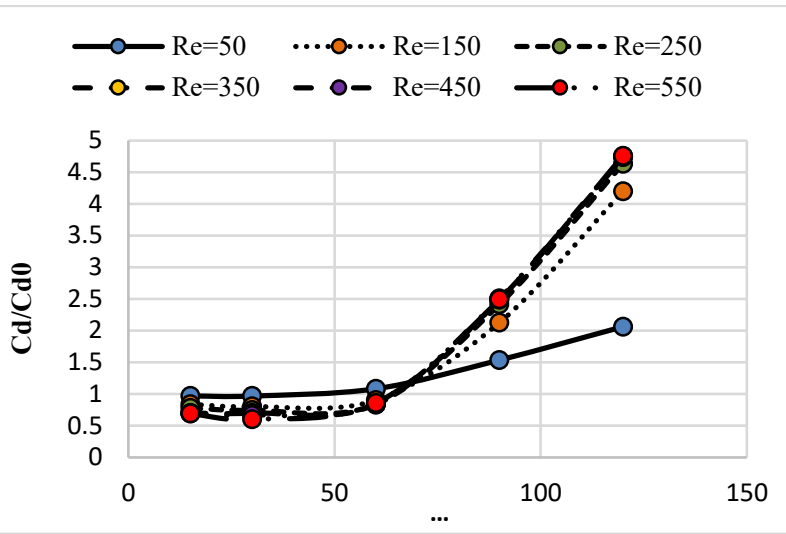

Figure 10. The ratio of total drag coefficient for cylinder and splitter plate assembly to that of bare cylinder

In Figure 11, the ratio of total heat transfer between fluid and cylinder and assembly to that of the bare cylinder is seen. In all Reynolds numbers, the diagrams dominate the unity, which is evident for the heat transfer rise. But by what angle among plates, this heat transfer will turn to its maximum amount? When the $\Theta$ rises, the plates' position seems to be more normal to the flow direction, which has two effects in essence. It provides a harder fluid strike to the plates, which intensifies the heat transfer. Also, it debilitates the flow between two plates downstream of the cylinder, which diminishes the heat convection between that region of the flow and the adjacent part of the circular cylinder. The $\mathrm{Re}=50$ diagram keeps its increasing tone as the angle grows. At high Reynolds numbers, such as $\mathrm{Re}=550$ or $\mathrm{Re}=250$, the vortex regime will again play an important role and, on $\theta=$ $30^{\circ}$ it decreases the heat convection the most, and on $\theta=60^{\circ}$ increases the heat convection the least. After $\theta=$ $60^{\circ}$ there is an area of heat transfer drop. Thus, for high Reynolds numbers, $\theta=60^{\circ}$ is chosen as the optimum point.

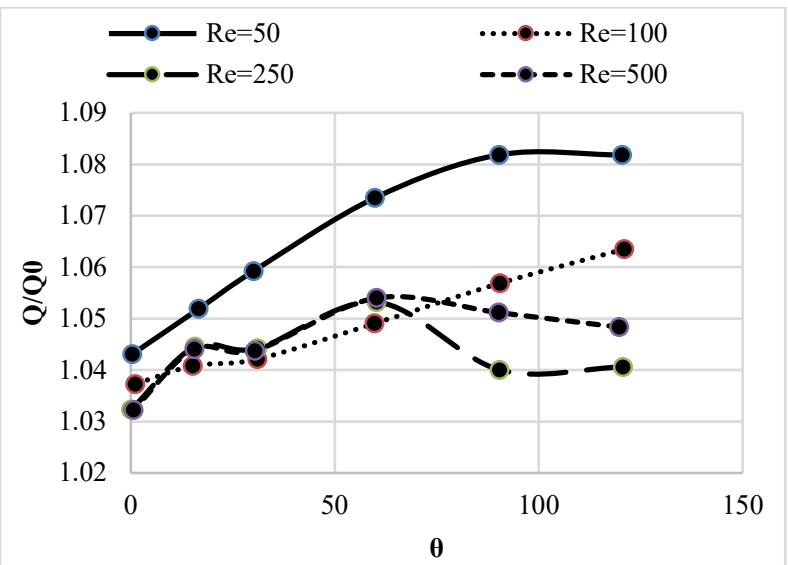

Figure 11. The ratio of total heat transferred from cylinder and splitter assembly to that of the bare cylinder.
In Figure 12, the Nusselt oscillations for the bare cylinder are noticed. Where in Figure 13, Nusselt waves are shown to be manipulated by a two-branched splitter plate. From this perspective, the angles $\theta=120^{\circ}$ and $\theta=90^{\circ}$ are totally rejected to be used for they have increased the oscillation amplitude by a significant amount. The minimum oscillation amplitude seems to happen at $\theta=30^{\circ}$.

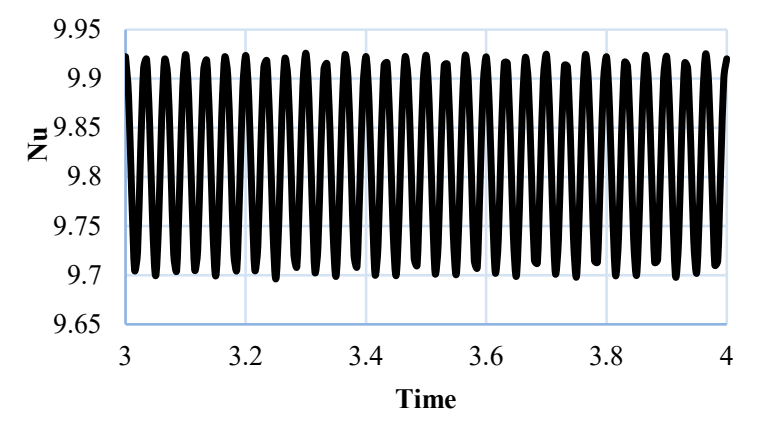

Figure 12. Nusselt oscillations for the bare cylinder at $R e=350$

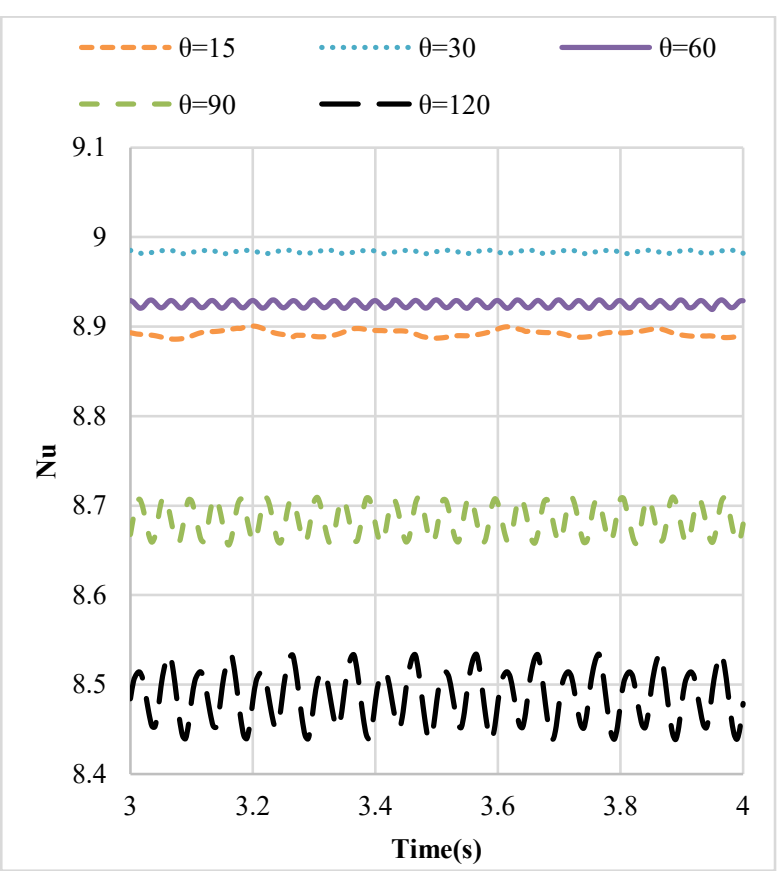

Figure 13. Nusselt Oscillations for the Cylinder bearing two branch splitter plate at $R e=350$

Here, the controlling parameter will turn to $n=l / D$ the dimensionless length, which is the measure of the length of splitter plate to the cylinder diameter. Figure 14, deals with the ratio of the drag coefficient for cylinder and splitter assembly to that of the bare cylinder. It is seen that at low Reynolds number, the ratio is closest to unity and the reason is a weakness of viscous agents and the pale effect of vortex shedding. For the splitter's main effect is to manipulate the vortex regime and where there is none, no significant effect on flow parameters is recognized due to splitter's presence. As the Reynolds grows, a point of minimum takes shape, which is pale in $\mathrm{Re}=150$ and $\mathrm{Re}=250$ but clear in higher Reynolds numbers. This point is in the region $0.75 \leq n \leq$ 1.00 and the reason, as previously stated might be related to the unavailability of the cylinder by the vortices. 


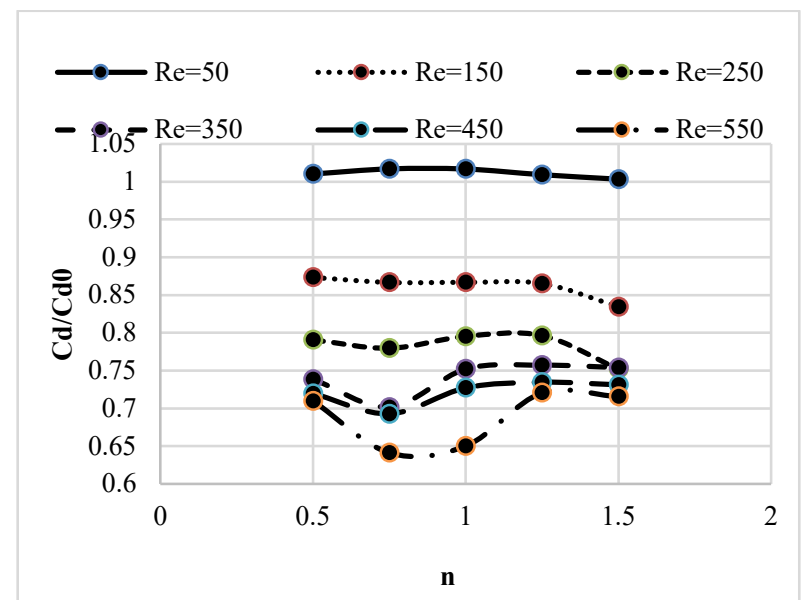

Figure 14. The ratio of drag coefficient of the cylinder with double branch splitter to that of the bare cylinder in various $R e$ and $n$ conditions.

It is seen that the diagram is close to unity at low $\operatorname{Re}(50$ and 150), and the reason was discussed before. When Reynolds number grows and viscous forces fortify, the vortex regime gets more robust, and a point of minimum for mean Nusselt number occurs. It is in the region of $0.75 \leq$ $n \leq 0.5$ and is likely to be closer to $n=0.75$. This issue is attributed to the low availability of the cylinder to the vortex, as clarified before. The correspondence between Nusselt and drag coefficient is in similarity with the Reynolds analogy.

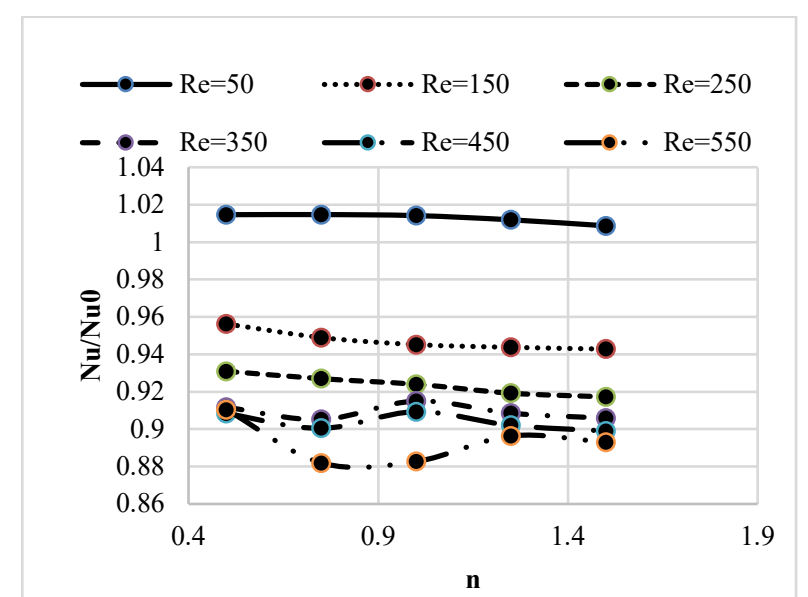

Figure 15. Nusselt Ratio between cylinder bearing double branch splitter plate and bare cylinder

As it is seen in Figure 15, when $\theta=30^{\circ}$, total drag was decreased by the double branched splitter plate. Because Figure 15 have been accomplished with $\theta=30^{\circ}$, the diagrams are expected to lie beneath the unity. It is proven that when the Reynolds number chooses low amounts, no specific effect is witnessed, and the diagram for $R e=50$ is most close to unity. All diagrams maintain their decreasing tone throughout various dimensionless lengths. When $\mathrm{n}=1.25$, the diagrams, stopped their diminishing trend and chose a more horizontal position. This means a further increase in the dimensionless size will not be beneficial. So, $\mathrm{n}=1.50$ seems the best according to total drag reduction.

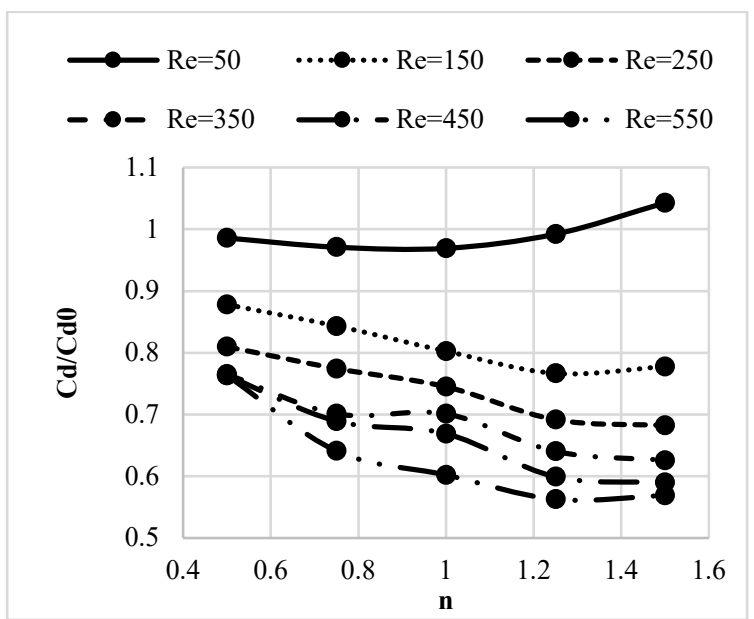

Figure 16. The ratio of total drag coefficient of the cylinder with double branch splitter to that of bare cylinder

The total heat transfer from the cylinder and splitter assembly to that of the bare cylinder is shown in Figure 17. The splitter plate could be counted as a fin. This is the main reason the diagrams maintain their increasing tone. This effect in low Reynolds number is the primary agent due to the weak development of viscous forces. When the Reynolds grows enough, this diminutive effect will weaken the heat transfer from the cylinder. The diminutive results are most potent in $0.75 \leq n \leq 1.00$ and hence shows up as a local minimum in the diagram. Overall, as it is clear from Figure 17 , no optimal dimensionless length could be chosen for this part; though there are local peaks, the growth of $\mathrm{n}$ ratio will increase the heat transfer area and its amount.

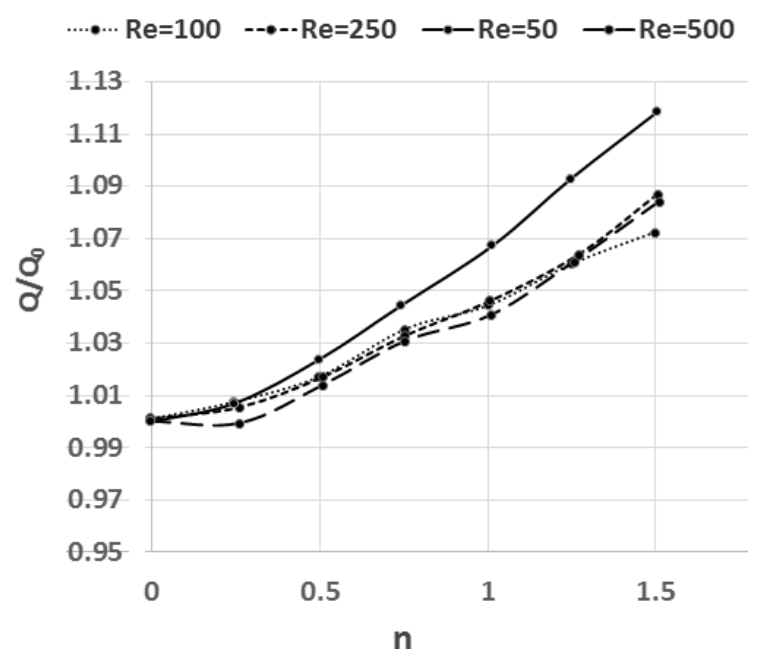

Figure 17. Ratio of total heat transferred from cylinder and splitter assembly to that of bare cylinder

Oscillation in heat transfer will cause thermal strain, and the drag force oscillation gives rise to yielding stresses. Both effects will damage the cylinder. So, it is useful to attempt minimizing the oscillation amplitude by any means. In Figure 18, the oscillation of the Nusselt number is shown. For $\mathrm{n}=0.5$, the oscillation amplitude has risen to a tremendous amount. On the other hand, $n=1.25$ and $n=1.50$ have successfully suppressed the amplitude and seem to be the best choices according to oscillation considerations. 


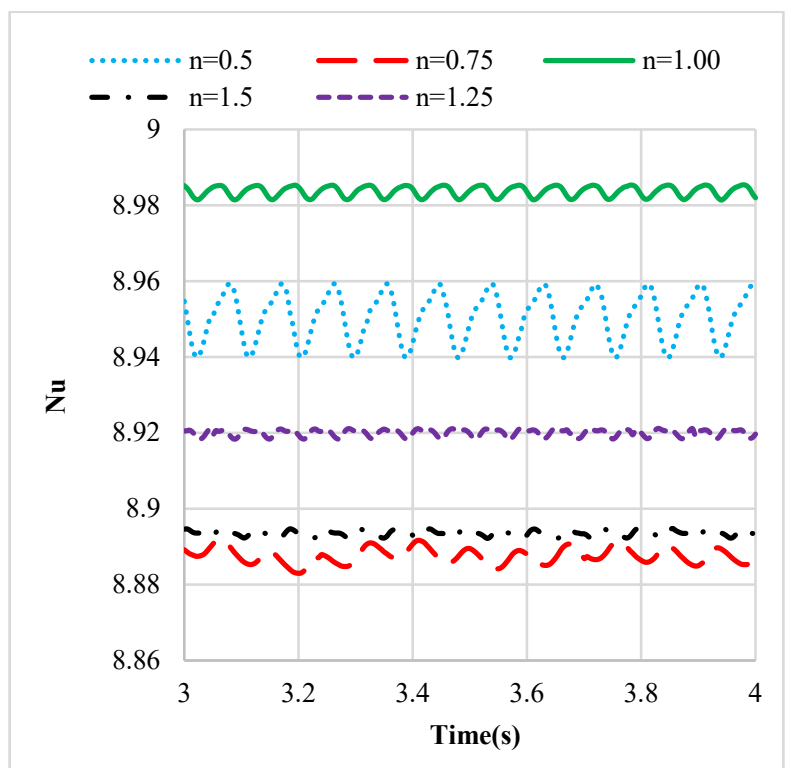

Figure 18. Nusselt Oscillations for the Cylinder bearing two branch splitter plate at $R e=350$

\section{Conclusion}

In this work, the double branch splitter plate was introduced, and various effect of angle among plates $\theta$ as well as dimensionless length $n$ of the splitter was investigated. The results demonstrate that when Reynolds number is low (around 50 or so), the use of a splitter plate makes no sense to heat transfer and drag. When Reynolds number grows to higher values $(\mathrm{Re}=550$ or so) among different angles, $\theta=30^{\circ}$ creates the least amount of drag for considering cylinder only, and $\theta=30^{\circ}$ also creates the least

drag on all cylinders and assembly. $\theta=30^{\circ}$ also makes the most drop in heat transfer considering cylinder only but for the entire assembly has it most heat transferred when $\theta=$ $60^{\circ}$. Angles more than $60^{\circ}$ are not recommended according to a dramatic increase in drag force $\theta=30^{\circ}$ also dampens the Nusselt oscillations the most, which will prevent rising yield stresses and thermal strain. The dimensionless length $\mathrm{n}=0.75$ (which is the ratio of splitter length to cylinder diameter) will cause the least drag as well as heat transfer, regarding the cylinder alone, and I the case of the entire assembly $n=1.25$ suits for the drag considerations but no optimal length could be introduced for heat transfer. The lengths $n=1.25$ and $n=1.5$ were found to diminish the oscillation amplitude more than other lengths being examined.

\section{Nomenclature}

$D$

$L$

$\theta$

$\rho$

$\mu$

$C_{P}$

$\Phi$

$P_{\text {out }}$

$T_{\text {in }}$

$T_{\text {cyl }}$

$\mathrm{m} / \mathrm{s}$
$\mathrm{cm}$
$\mathrm{cm}$
$\mathrm{degree}$
$\mathrm{kg} / \mathrm{m}^{\wedge} 3$
$\mathrm{~kg} \cdot \mathrm{m}^{\wedge}-1 \cdot \mathrm{s}^{\wedge}-1$
$\mathrm{~J} \cdot \mathrm{kg}-1 \cdot \mathrm{K}-1$
$\mathrm{~kg} \cdot \mathrm{m}^{\wedge} 2 \cdot \mathrm{s}^{\wedge} 2$
$\mathrm{~atm}$
$\mathrm{k}$
$\mathrm{k}$

Velocity

Cylinder Diameter

Cylinder Height

Splitter plates angle

Density

Static viscosity

Specific heat capacity

Dissipation term.

Outlet Pressure

Inlet Temperature

Cylinder Temperature

\section{References}

[1] T. Adibi, S. E. Razavi, and O. Adibi, "A Characteristic-based Numerical Simulation of Water-titanium Dioxide Nano-fluid in Closed Domains," (in en), International Journal of Engineering, vol. 33, no. 1, pp. 158-163, 2020, doi: 10.5829/ije.2020.33.01a.18.

[2] M. Y. Malik, M. Awais, T. Salahuddin, A. Hussain, and S. Bilal, "Mixed-convection boundary-layer flow of Sisko fluid along a stretching cylinder in a thermally strati ed medium," Scientia Iranica, vol. 23, no. 6, pp. 2587-2594, 2016, doi: 10.24200/sci.2016.3968.

[3] R. Bayat and A. B. Rahimi, "Numerical solution to N-S equations in the case of unsteady axisymmetric stagnation-point flow on a vertical circular cylinder with mixed convection heat transfer," Scientia Iranica, vol. 25, no. 4, pp. 2130-2143, 2018, doi: 10.24200/sci.2017.4347.

[4] F. Gu, J. S. Wang, X. Q. Qiao, and Z. Huang, "Pressure distribution, fluctuating forces and vortex shedding behavior of circular cylinder with rotatable splitter plates," Journal of Fluids and Structures, vol. 28, pp. 263-278, 2012/01/01/ 2012, doi: https://doi.org/10.1016/j.jfluidstructs.2011.11.0 $\underline{05}$.
[5] A. Igbalajobi, J. F. McClean, D. Sumner, and D. J. Bergstrom, "The effect of a wake-mounted splitter plate on the flow around a surfacemounted finite-height circular cylinder," Journal of Fluids and Structures, vol. 37, pp. 185-200, 2013/02/01/ 2013, doi: https://doi.org/10.1016/j.jfluidstructs.2012.10.0 $\underline{01}$.

[6] N. Boisaubert and A. Texier, "Effect of a splitter plate on the near-wake development of a semicircular cylinder," Experimental Thermal and Fluid Science, vol. 16, no. 1, pp. 100-111, 1998/01/01/ 1998, doi: https://doi.org/10.1016/S0894-1777(97)100097.

[7] S. Shukla, R. N. Govardhan, and J. H. Arakeri, "Flow over a cylinder with a hinged-splitter plate," Journal of Fluids and Structures, vol. 25, no. 4, pp. 713-720, 2009/05/01/ 2009, doi: https://doi.org/10.1016/j.jfluidstructs.2008.11.0 $\underline{04}$.

[8] R. Abdi, N. Rezazadeh, and M. Abdi, "Reduction of fluid forces and vortex shedding frequency of a circular cylinder using rigid splitter plates," European Journal of Computational Mechanics, vol. 26, no. 3, pp. 225-244, 2017/05/04 2017, doi: $10.1080 / 17797179.2017 .1306826$. 
[9] S. Dai, B. A. Younis, H. Zhang, and C. Guo, "Prediction of vortex shedding suppression from circular cylinders at high Reynolds number using base splitter plates," Journal of Wind Engineering and Industrial Aerodynamics, vol. 182, pp. 115-127, 2018/11/01/ 2018, doi: https://doi.org/10.1016/j.jweia.2018.09.006.

[10] D.-L. Gao, G.-B. Chen, Y.-W. Huang, W.-L. Chen, and H. Li, "Flow characteristics of a fixed circular cylinder with an upstream splitter plate: On the plate-length sensitivity," Experimental Thermal and Fluid Science, vol. 117, p. 110135, 2020/09/01/ 2020, doi: https://doi.org/10.1016/j.expthermflusci.2020.1 10135 .

[11] S. Tiwari, D. Chakraborty, G. Biswas, and P. K. Panigrahi, "Numerical prediction of flow and heat transfer in a channel in the presence of a built-in circular tube with and without an integral wake splitter," International Journal of Heat and Mass Transfer, vol. 48, no. 2, pp. 439-453, 2005/01/01/ 2005, doi: https://doi.org/10.1016/j.ijheatmasstransfer.200 4.09.003.

[12] H. Akilli, B. Sahin, and N. Filiz Tumen, "Suppression of vortex shedding of circular cylinder in shallow water by a splitter plate," Flow Measurement and Instrumentation, vol. 16, no. 4, pp. 211-219, 2005/08/01/ 2005, doi: https://doi.org/10.1016/j.flowmeasinst.2005.04. $\underline{004}$.

[13] X. An, B. Song, W. Tian, and C. Ma, "Numerical Research of Flow past a Circular Cylinder with Splitter Plate at a Subcritical Reynolds Number Region," Journal of Shanghai Jiaotong University (Science), journal article vol. 24, no. 1, pp. 113-121, February 01 2019, doi: 10.1007/s12204-019-2045-y.

[14] T. R. Sahu, M. Furquan, Y. Jaiswal, and S. Mittal, "Flow-induced vibration of a circular cylinder with rigid splitter plate," Journal of Fluids and Structures, 2019/03/25/ 2019, doi: https://doi.org/10.1016/j.jfluidstructs.2019.03.0 $\underline{15}$.

[15] A. Ghiasi, "Numerical study on flow over a confined oscillating cylinder with a splitter plate," International Journal of Numerical Methods for Heat \&amp; Fluid Flow, vol. 29, no. 5, pp. 1629-1646, 2019, doi: 10.1108/HFF-062018-0286.

[16] R. Mishra, S. S. Kulkarni, R. Bhardwaj, and M. C. Thompson, "Response of a linear viscoelastic splitter plate attached to a cylinder in laminar flow," Journal of Fluids and Structures, vol. 87, pp. 284-301, 2019/05/01/ 2019, doi: https://doi.org/10.1016/j.jfluidstructs.2019.03.0 26.

[17] M. K. Chauhan, S. Dutta, B. S. More, and B. K. Gandhi, "Experimental investigation of flow over a square cylinder with an attached splitter plate at intermediate reynolds number," Journal of Fluids and Structures, vol. 76, pp. 319-335, 2018/01/01/ 2018, doi: https://doi.org/10.1016/j.jfluidstructs.2017.10.0 12.
[18] G. R. S. Assi and P. W. Bearman, "Transverse galloping of circular cylinders fitted with solid and slotted splitter plates," Journal of Fluids and Structures, vol. 54, pp. 263-280, 2015/04/01/ 2015, doi: https://doi.org/10.1016/j.jfluidstructs.2014.11.0 $\underline{05}$.

[19] G. Ghassabi and M. kahrom, "Heat Transfer Enhancement of a Flat Plate Boundary Layer Distributed by a Square Cylinder: Particle Image Velocimetry and Temperature-Sensitive Paint Measurements and Proper Orthogonal Decomposition Analysis," International Journal of Engineering, vol. 31, no. 11, pp. 1962-1971, 2018. [Online]. Available: http://www.ije.ir/article_82253_411facead24d1 c6e7b4b94b5395a3a37.pdf.

[20] T. Adibi and O. Adibi, "Laminar Forced Convection Simulation at Different Boundary Conditions with Averaging Scheme (Numerical and Theoretical Research)," $M M E P$, vol. 6, no. 4, pp. 519-526, 2019/12/24/ 2019, doi: 10.18280/mmep.060406.

[21] E. Sciubba, "Flow Exergy as a Lagrangian for the Navier-Stokes Equations for Incompressible Flow," (in en), International Journal of Thermodynamics, vol. 7, no. 3, pp. 115-122, 2004/09/01/ 2004. [Online]. Available: https://dergipark.org.tr/en/pub/ijot/76673.

[22] V. R. Manthena and G. D. Kedar, "Mathematical Modeling of Thermoelastic State of a Functionally Graded Thermally Sensitive Thick Hollow Cylinder With Internal Heat Generation," (in en), International Journal of Thermodynamics, vol. 21, no. 4, pp. 202-212, 2018/12/04/ 2018, doi: 10.5541/ijot.434180.

[23] S. E. Razavi and T. Adibi, "A novel multidimensional characteristic modeling of incompressible convective heat transfer," Journal of Applied Fluid Mechanics, vol. 9, no. 4, pp. 1135-1146, 2016 2016, doi: 10.18869/acadpub.jafm.68.228.24295.

[24] T. Adibi, "Three-dimensional characteristic approach for incompressible thermo-flows and influence of artificial compressibility parameter," (in en), Journal of Computational \& Applied Research in Mechanical Engineering (JCARME), vol. 8, no. 2, pp. 223-234, 02/01 2019, doi: 10.22061/jcarme.2018.2032.1178.

[25] T. Adibi, O. Adibi, and S. E. Razavi, "A characteristic-based solution of forced and free convection in closed domains with emphasis on various fluids " International Journal of Engineering, vol. 32, no. 10, pp. 1679-1685, 2019, doi: 10.5829/ije.2019.32.11b.20.

[26] T. Adibi and S. E. Razavi, "A new characteristic approach for incompressible thermo-flow in Cartesian and non-Cartesian grids," International Journal for Numerical Methods in Fluids, vol. 79, no. 8, pp. 371-393, 2015, doi: 10.1002/fld.4053.

[27] S. W. Churchill and M. Bernstein, "a correlation coefficient for forced convection from gases and liquids to a circular cylinder in cross flow," $J$. Heat Transfer, vol. 99, pp. 300-306, 1977. 
[28] Seyed Esmail Razavi, Vahid Farhangmehr, and F. Barar, " Impact of a Splitter Plate on Flow and Heat Transfer Around Circular Cylinder at Low Reynolds Numbers," Journal of Applied Sciences, vol. 8, pp. 1286-1292, 2008, doi: $10.3923 /$ jas.2008.1286.1292
[29] S. E. Razavi, T. Adibi, and S. Faramarzi, "Impact of inclined and perforated baffles on the laminar thermo-flow behavior in rectangular channels," SN Applied Sciences, vol. 2, no. 2, p. 284, 2020/01/29 2020, doi: 10.1007/s42452-0202078-8. 